\title{
The magic of improved crystal technology coupled with better resolution: Novel imaging findings may promise improved disease detection
}

\author{
Vikas Veeranna, $M D{ }^{a}$ and Andrew M. Freeman, MD, FACC ${ }^{b}$ \\ a New England Heart and Vascular Institute, Manchester \\ b Division of Cardiology, Department of Medicine, National Jewish Health, Denver
}

Received Sep 9, 2020; accepted Sep 9, 2020

doi: $10.1007 / \mathrm{s} 12350-020-02380-1$

See related article, pp. 320-327

Myocardial perfusion imaging (MPI) with singlephoton emission tomography (SPECT) is widely performed for the diagnosis and management of patients with suspected or known coronary artery disease (CAD). ${ }^{1,2}$ Non-invasive assessment with MPI not only has an excellent diagnostic and prognostic accuracy but also provides good insight into the cardiac function. Over the past few years, there has been significant advances made in terms of SPECT equipment, with newer scanners dedicated to cardiac imaging utilizing solid-state crystals and novel collimator designs. ${ }^{1}$ Newer cardiac-dedicated camera designs vary in whether Sodium Iodide (NaI), Cesium Idodie (CSI) or Cadmium Zinc Telluride (CZT) solid-state detectors are used and also the number and type of scanning or stationary detectors. ${ }^{2}$ With the CZT camera there is simultaneous improvement in image resolution (up to two times higher) due to improved energy resolution of the solidstate crystals, the sensitivity being more than five times higher due to the collimator, and improved spatial resolution in comparison to the conventional SPECT cameras. ${ }^{3}$ These innovations have facilitated significant improvement in image quality with reductions in

Reprint requests: Andrew M. Freeman, MD, FACC, Division of Cardiology, Department of Medicine, National Jewish Health, Denver; andrew@docandrew.com

J Nucl Cardiol 2021;28:328-30.

$1071-3581 / \$ 34.00$

Copyright (c) 2020 American Society of Nuclear Cardiology. imaging time as well as radiation dose while maintaining high levels of diagnostic accuracy. ${ }^{4}$

The D.SPECT camera (Spectrum Dynamics, Caesarea, Israel) involves the use of nine rotating columns associated with a wide-angle square hole collimator made of Tungsten with CZT crystals aligned behind each collimator and the columns are arranged in a curved configuration to conform to the shape of the left side of the subject's chest. ${ }^{5}$ This collimator has greater than 10 times the count sensitivity and a high level of spatial resolution that can be reached owing to a specific reconstruction method. ${ }^{5}$ Better delineation of the myocardial walls due to higher spatial resolution and more focused imaging of the heart with the reduction of attenuation artifacts due to rotational parallel-hole collimation have led to studies showing that the MPI acquired with newer cardiac dedicated cameras with CZT have a diagnostic accuracy significantly higher than that obtained with standard SPECT. ${ }^{1,4,6}$

Despite the advances in SPECT MPI, there are certain limitations irrespective of the technology. An underestimation of the assessment of high-risk anatomic $\mathrm{CAD}$, especially due to balanced ischemia, has been well described with SPECT MPI. ${ }^{7}$ The careful assessment of other variables derived with SPECT MPI including transient ischemic dilation (TID), drop in ejection fraction (EF) with stress or low EF, abnormal wall motion, increased right ventricular and lung uptake of the tracer in addition to the clinical and stress information have assisted in better recognition of high risk $\mathrm{CAD}^{7,8}$ However, the data regarding TID reference levels with newer cardiac dedicated cameras are yet to be established firmly. ${ }^{9,10}$ The search for other imaging parameters which can further help in the interpretation of SPECT MPI and better risk stratification is ongoing. 
In the current issue of the Journal, Vadi et al. describe an interesting observation based on the presence of reversible perfusion pattern in hypertrophic papillary muscles seen on myocardial perfusion imaging using the aforementioned D SPECT camera. (This article) While the spatial resolution from the older generation conventional cameras may not allow for visualization of papillary muscles (PM), the improved spatial resolution with newer generation cameras probably allows for a better assessment of PM tracer uptake and may need to be considered during image interpretation. A similar finding of PM ischemia has been documented with positron emission tomography (PET) MPI owing to its improved spatial resolution in comparison to SPECT MPI. ${ }^{11,12}$

The left ventricular (LV) PMs are pillar-like muscles arising from the left ventricular wall. Typically there are two PMs, the anterolateral and the posteromedial, aiding in the proper functioning of the mitral valve. ${ }^{13}$ The blood supply of the PMs also appears to be variable. While the anterolateral PM is often perfused by both 1st obtuse marginal branch of the left circumflex and the 1st diagonal branch of the left anterior descending (LAD) coronary artery, the posteromedial PM usually has a single coronary supply either the right coronary artery or the third obtuse marginal branch. ${ }^{14}$

In this present study, a total of just 6 patients with chronic hypertension were identified having PM ischemia based on presence of increased tracer uptake in the region of PM in the LV cavity on resting images when compared to stress images on either pharmacologic or exercise treadmill myocardial perfusion imaging. The presence of hypertrophy involving the PM was confirmed using mostly cardiac magnetic resonance imaging and echocardiogram. The presence of PM ischemia was also associated with the presence of ischemia ranging from 5\% to $12 \%$ and in their data was predominantly associated with perfusion abnormalities in the LAD territory. PM ischemia is known to coexist with regional ischemia but more often accompanied by ischemia in the left circumflex region based on the heterogeneous nature of its blood supply. Similar association of PM ischemia with perfusion abnormalities was noted in study by Nakao et al., using high-resolution cine imaging of Nitrogen 13 ammonia PET scan, PM ischemia was identified in close to $12 \%$ of the patients and was also shown to have higher burden of regional ischemia. $^{11}$

Further, 2 out of 6 patients had TID with an accompanying drop in LVEF post-stress. Although the authors could not confirm the presence of obstructive $\mathrm{CAD}$ in all the patients, they postulated these changes may be related to early signs of endomyocardial ischemia. As the endocardial papillary muscles derive their arterial supply from the distal and deeper coronary branches, especially in the context of a hypertrophied papillary muscle, the ischemic response during myocardial perfusion imaging was evident with a CZT camera with its improved spatial resolution.

These findings may be further supported by data from a PET MPI study by Nakao et al. suggesting that reduced myocardial blood flow reserve was seen in patients with papillary muscle ischemia irrespective of the CAD status implicating significant microvascular dysfunction contributing to this pattern. ${ }^{11}$ While the authors do not have information about follow-up for adverse cardiovascular events, the presence of PM ischemia and its association with perfusion abnormalities as well as reduced myocardial blood flow reserve have been associated with increased adverse cardiovascular events. ${ }^{11}$

Does this finding observed in six patients with hypertrophied PMs in this study by Vadi et al. really translate to an additional parameter, similar to TID? Not quite yet: additional validations with deeper follow up for outcomes along with larger samples sizes are needed. Nevertheless, this is a novel finding that deserves further evaluation with dedicated studies correlating with presence of obstructive CAD as well as adverse cardiovascular events.

\section{Disclosure}

A. Freeman does non promotional speaking for BoehringerIngelheim, consulting for Boehringer-Ingelheim, Actelion, The Medicines Company, Regeneron V. Veeranna has nothing to disclose.

\section{References}

1. Abbott BG, Case JA, Dorbala S, et al. Contemporary cardiac SPECT imaging-innovations and best practices: An information statement from the American Society of Nuclear Cardiology. J Nucl Cardiol. 2018;25:1847-60.

2. Dorbala S, Ananthasubramaniam K, Armstrong IS, et al. Single photon emission computed tomography (SPECT) myocardial perfusion imaging guidelines: Instrumentation, acquisition, processing, and interpretation. J Nucl Cardiol. 2018;25:1784-846.

3. Imbert L, Marie PY. CZT cameras: A technological jump for myocardial perfusion SPECT. J Nucl Cardiol. 2016;23:894-6.

4. Sharir T, Slomka PJ, Hayes SW, et al. Multicenter trial of highspeed versus conventional single-photon emission computed tomography imaging: Quantitative results of myocardial perfusion and left ventricular function. J Am Coll Cardiol. 2010;55:196574.

5. Gambhir SS, Berman DS, Ziffer J, et al. A novel high-sensitivity rapid-acquisition single-photon cardiac imaging camera. J Nucl Med. 2009;50:635-43.

6. Nakazato R, Slomka PJ, Fish M, et al. Quantitative high-efficiency cadmium-zinc-telluride SPECT with dedicated parallel-hole 
collimation system in obese patients: Results of a multi-center study. J Nucl Cardiol. 2015;22:266-75.

7. Nakanishi R, Gransar H, Slomka P, et al. Predictors of high-risk coronary artery disease in subjects with normal SPECT myocardial perfusion imaging. J Nucl Cardiol. 2016;23:530-41.

8. Alama M, Labos C, Emery H, et al. Diagnostic and prognostic significance of transient ischemic dilation (TID) in myocardial perfusion imaging: A systematic review and meta-analysis. J Nucl Cardiol. 2018;25:724-37.

9. Reyes E. Refining practice: TID metrics for CZT systems. J Nucl Cardiol. 2020;27:1190-2.

10. Hu LH, Sharir T, Miller RJH, et al. Upper reference limits of transient ischemic dilation ratio for different protocols on newgeneration cadmium zinc telluride cameras: A report from REFINE SPECT registry. J Nucl Cardiol. 2020;27:1180-9.
11. Nakao R, Nagao M, Yamamoto A, et al. Papillary muscle ischemia on high-resolution cine imaging of nitrogen-13 ammonia positron emission tomography: Association with myocardial flow reserve and prognosis in coronary artery disease. Clinical nuclear medicine. 2020. https://doi.org/10.1007/s12350-020-02231-z.

12. Steinmetz AP, Maisey MN, Hardoff R. Patterns of papillary muscle ischemia in myocardial PET. Clin Nucl Med. 2004;29:1-4.

13. Rich NL, Khan YS. Anatomy, thorax, heart papillary muscles. Treasure Island: StatPearls; 2020.

14. Voci P, Bilotta F, Caretta Q, Mercanti C, Marino B. Papillary muscle perfusion pattern. A hypothesis for ischemic papillary muscle dysfunction. Circulation. 1995;91:1714-8.

Publisher's Note Springer Nature remains neutral with regard to jurisdictional claims in published maps and institutional affiliations. 